\title{
Optimizing the Configuration of Web Service Monitors
}

\author{
Garth Heward ${ }^{1}$, Jun Han ${ }^{1}$, Ingo Müller ${ }^{1}$, \\ Jean-Guy Schneider ${ }^{1}$, and Steve Versteeg ${ }^{2}$ \\ 1 Swinburne University of Technology, Hawthorn, Victoria, Australia \\ 2 CA Labs, Melbourne, Victoria, Australia \\ \{gheward, jhan, imueller, jschneider\}@swin.edu.au, \\ Steve.Versteeg@ca.com
}

\begin{abstract}
Service monitoring is required for meeting regulatory requirements and verifying compliance to Service Level Agreements (SLAs). As such, monitoring is an essential part of web service-based systems. However, service monitoring comes with a cost, including an impact on the quality of monitored services and systems. To deliver the best value to a service provider, it is important to balance meeting monitoring requirements and reducing monitoring impacts. We introduce a novel approach to configuring the web service monitors deployed in a system so that they provide an adequate level of monitoring but with minimized quality impacts, delivering the best value proposition in terms of monitoring benefits and costs. We use a prototype system to demonstrate that by optimizing a web service monitoring system, we can reduce the impact of a set of deployed web service monitors by up to two thirds.
\end{abstract}

Keywords: Web Services, Monitoring, Monitoring Optimization.

\section{Introduction}

Web service providers give quality guarantees for their services, so that consumers know the expected Quality of Service (QoS) of services prior to using them. To demonstrate that they have met these guarantees, providers monitor their services. Whilst achieving this goal, service monitoring creates a problem because the monitoring itself reduces the quality of the web services. This impact has been demonstrated to be as much as $40 \%$ (for response time) for a single monitor 1]. As such, there is a conflict between the goal of performing monitoring, and the goal of maintaining acceptable quality levels.

To meet these conflicting goals, a web service provider should carefully select what monitoring is performed in the system in order to meet their monitoring obligations without violating any quality guarantees or requirements. Achieving a balance between meeting monitoring requirements and achieving required quality levels by manual configuration is difficult, time-consuming, and unverified for optimality. We propose an automated approach, which allows a service provider to discover an optimal monitoring configuration with less human effort. 
Methods exist for optimizing the performance of web service systems through selective execution or load balancing, e.g. 223]. Whilst highlighting the need to optimize the performance of web service systems, none of these techniques relate to the management of deployed service monitors. Only Baresi and Guinea [4] discuss the configuration of web service monitors in order to achieve a trade-off between performance and monitoring. However, they only go as far as manually assigning a resolution of monitoring to each individual service in the system.

We introduce a novel approach that takes as inputs the monitoring requirements (benefits) and the monitoring impacts on system performance (costs), and determines an optimal monitoring configuration that delivers the best value by trading-off these benefits and costs. It considers the quality aspects and monitoring levels for each monitor and service. The optimal monitoring configuration consists of which monitors should be enabled, for what measures of quality, and at what sampling rates, in order to meet monitoring requirements whilst not reducing delivered qualities of service to unacceptable levels.

We demonstrate the benefits (the performance and utility increases) of applying our approach to the deployed monitors of a web service system. We have shown that in the best case, the web services under an optimal monitoring configuration had a response time impact just one third of the impact that a standard monitoring configuration had on the same services.

In Section 2, we introduce our approach to automated monitoring optimization. In Section 3, we discuss the experiments conducted to demonstrate the possible performance benefits of our approach.

\section{Monitoring Optimization}

As discussed above, to optimize the configuration of deployed web service monitors, their impact on system performance must be minimised, whilst meeting monitoring requirements. As such, the optimization problem is to find a monitoring configuration that gives the maximum value (utility) in terms of performance impact (costs) and monitoring coverage (benefits). Figure 1 shows a framework illustrating our approach to monitoring optimization, annotated with the ordering of activities of the optimization process. In step 1, the set of deployed monitors is identified based on IT records, and then the Enumerator generates all the possible monitoring configurations for this set of monitors. In step $\mathbf{2}$, the performance impacts of each monitor are identified from IT Records or benchmarking, and the Impact Analyser derives the total performance impact of each possible monitoring configuration. In step 3, the monitoring requirements and associated penalties for not meeting them are identified from analysing SLAs, policies and laws, and the Requirements Analyser transforms requirements into a set of utility functions that define the benefit gained from monitoring a quality whilst a specific level of performance is being achieved. In step 4, the Optimizer uses the set of utility functions from the Requirements Analyser to score all monitoring configurations with impacts from the Impact Analyser, and the monitoring configuration that yields the highest utility is selected and applied to the monitoring system. Each framework component is described below in more detail. 


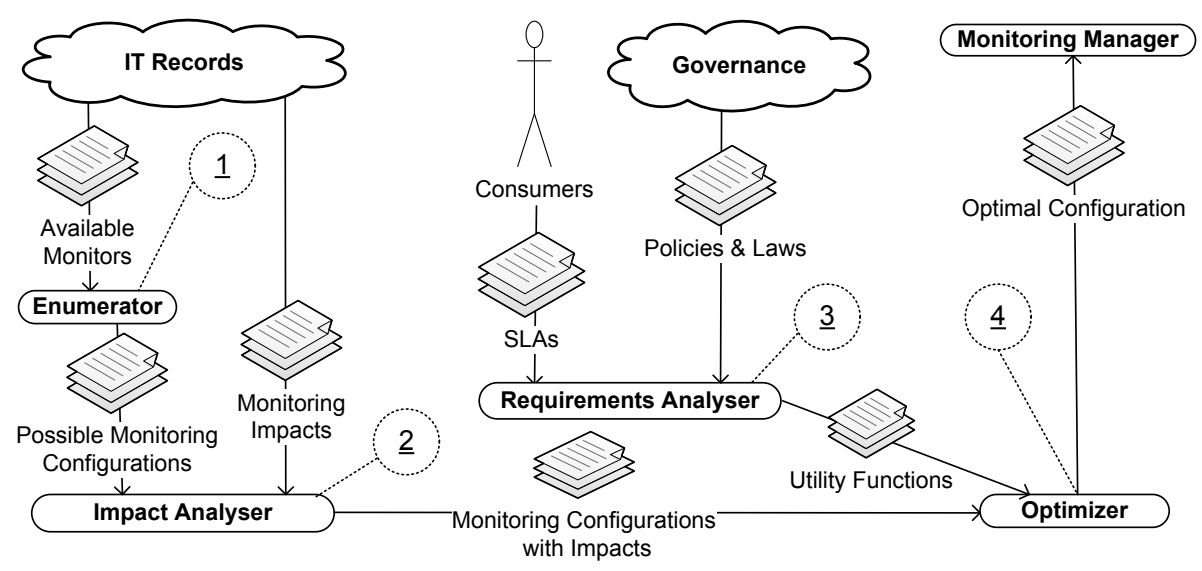

Fig. 1. Monitoring Optimization Framework

Enumerator. The Enumerator takes as input the set of available monitors, and outputs the set of all possible monitoring configurations.

A monitor can monitor one or more quality types of one or more services, simultaneously or separately. We refer to the set of all quality types of a system as $Q$, and the set of all services as $S$. A monitoring capability, mcap $=(m, s, q) \mapsto$ $S R$ represents the ability of a monitor $m \in M$ to monitor a quality $q \in Q$ of a service $s \in S$ at a sampling rate $s r \in S R$. A monitoring setting, $m s=$ $((m, s, q) \mapsto s r, s r \in \operatorname{mcap}(m, s, q)$ represents a monitoring capability set at a particular sampling rate $s r$. $M S$ represents the set of all possible monitoring settings. A set of monitoring settings obtained by assigning every monitoring capability to a sampling rate is called a monitoring configuration, $m c \in M C$ and $m c=\{(m, s, q) \mapsto s r \in \operatorname{mcap}(m, s, q) \mid \forall(m, s, q) \in M C A P\}$, where $M C A P$ is the set of all the deployed monitoring capabilities in the system.

For example, if we have monitor $m_{1}$ capable of monitoring quality $q_{1}$ of service $s_{1}$ at sampling rates of $0,0.5$, or 1.0 , and monitor $m_{2}$, capable of monitoring quality $q_{1}$ and $q_{2}$ of service $s_{1}$ at sampling rates of 0 or 1.0 , the three monitoring capabilities are $\left\{\left(m_{1}, s_{1}, q_{1}\right) \mapsto(0,0.5,1.0),\left(m_{2}, s_{1}, q_{1}\right) \mapsto(0,1.0),\left(m_{2}, s_{1}, q_{2}\right) \mapsto\right.$ $(0,1.0)\}$. One of the monitoring configurations is $m c=\left\{\left(m_{1}, s_{1}, q_{1}\right) \mapsto 0.5,\left(m_{2}\right.\right.$, $\left.\left.\left.s_{1}, q_{1}\right) \mapsto 0,\left(m_{2}, s_{1}, q_{2}\right) \mapsto 1.0\right\}\right\}$. The Enumerator generates all possible monitoring configurations $M C$ by stepping through all the allowable combinations of monitors, services, qualities and sampling rates. For the above example, the output from the Enumerator is presented in Table1, where a row corresponds to a monitoring capability, a column corresponds to a monitoring configuration, and a cell is the specific sampling rate of the corresponding monitoring capability in the relevant monitoring configuration. The monitoring configuration mentioned above is column $m c_{8}$ in Table 1 
Table 1. Monitoring Enumeration

\begin{tabular}{|c|c|c|c|c|c|c|c|c|c|c|c|c|}
\hline & $m c_{1}$ & $m c_{2}$ & $m c_{3}$ & $m c_{4}$ & $m c_{5}$ & $m c_{6}$ & $m c_{7}$ & $m c_{8}$ & $m c_{9}$ & $m c_{10}$ & $m c_{11}$ & $m c_{12}$ \\
\hline$m_{1} s_{1} q_{1}$ & 10 & 0.5 & 1.0 & 0 & $\int 0.5$ & $\int 1.0$ & 0 & 0.5 & (1.0) & 0 & 0.5 & 1.0 \\
\hline$m_{2} s_{1} q_{1}$ & 0 & 0 & 0 & $\{1.0$ & $\{1.0\}$ & $\{1.0\}$ & 0 & 0 & 0 & $\{1.0$ & 1.0 & $1.0\}$ \\
\hline$m_{2} s_{1} q_{2}$ & 0 & 0 & 0 & 0 & 0 & 0 & $(1.0)$ & (1.0) & (1.0) & $(1.0)$ & $1.0)$ & $1.0)$ \\
\hline
\end{tabular}

Impact Analyser. The Impact Analyser takes as input all possible monitoring configurations $M C$, all monitor setting impacts $M I$ and monitor overheads $M O$, and outputs the set of monitoring configurations with impacts $M C I$.

$i l$ denotes the impact level on a quality $q$ of a service $s,(s, q) \mapsto i l$. Impact level $i l \in I L$ is an impact as a percentage on the original quality. For example, the impact of $\left(\left(s_{1}, q_{1}\right) \mapsto 0.5\right)$ means the level of $q_{1}$ of $s_{1}$ is halved.

Monitoring has both overhead and monitor setting impacts. The overhead mo of a monitor $m$ on a service $s$ occurs whenever the monitor is in use, regardless of the activities of the monitor. Let $S Q$ be all the service-quality pairs of concern in the system, the overheads a monitor has on every relevant quality $q$ of every service $s$ are $m o=\{(s, q) \mapsto i l: I L \mid \forall(s, q) \in S Q\}$. For example, an interceptor $m_{1}$ that redirects messages of services $s_{1}$ and $s_{2}$ for analysis may reduce response time of all messages passing through it by $10 \%$, whether or not those messages are actually analysed. Let $M I S=S Q \rightarrow I L$, the set of all monitor overheads is the collection of all the monitors' overheads, $M O=\{m \mapsto \mathcal{P}(M I S) \mid \forall m \in M\}$.

The monitoring setting impacts $m s i$ represent the impacts that a monitoring setting $m s$ has on each quality $q$ of each service $s$ in the system. That is, $m s i=$ $\{(s, q) \mapsto i l: I L \mid \forall(s, q) \in S Q\}$. For example, IT records may show that when monitor $m_{1}$ is configured to measure $q_{1}$ of $s_{1}$ at sampling rate 0.5 , it reduces $q_{1}$ of services $s_{1}$ and $s_{2}$ by $10 \%$. The impacts of this monitor setting are $\left\{\left(s_{1}, q_{1}\right) \mapsto\right.$ $\left.0.1,\left(s_{2}, q_{1}\right) \mapsto 0.1\right\}$. The set of impacts of all possible monitor settings in $M S$ is: $M I=\{m s \mapsto m s i: \mathcal{P}(M I S) \mid \forall m s \in M S\}$.

The impact of a monitoring configuration $m c$ on quality $q$ of service $s$ is made up the following components: (1) the sum of the impacts of all monitoring settings on $q$ of $s: I_{s, q}(m c)=\sum_{m s \in m c} M I(m s)(s, q)$; and (2) the sum of all the monitors' overheads on $q$ of service $s: O_{s, q}(m c)=\sum_{m \in M} M O(m)(s, q) \times$ $i n U s e(m)$, where $i n U s e(m)=\left\{\begin{array}{l}1 \text { iff } \exists m c_{i} \in m c, m c_{i} . m=m, m c_{i} . s r>0 \\ 0 \text { iff } \nexists m c_{i} \in m c, m c_{i} . m=m, m c_{i} . s r>0\end{array}\right\}$. Therefore, $m c^{\prime}$ 's impacts on $q$ of $s$ are: $I M(m c)(s, q)=I_{s, q}(m c)+O_{s, q}(m c)$, and $m c$ 's impacts on all $(s, q)$ pairs are: $I M(m c)=\{(s, q) \mapsto I M(m c)(s, q) \mid \forall(s, q) \in S Q\}$. Finally, the set of all monitoring configurations with impacts (on service-quality pairs) is: $M C I=\{m c \mapsto I M(m c) \mid \forall m c \in M C\}$.

Requirements Analyser. The Requirements Analyser takes requirements from SLAs and other sources such as corporate governance as input, and outputs a set of utility functions representing the values of achieving those requirements.

We assume that requirements can be translated into statements that describe a fine for not meeting or not monitoring a QoS. For example, an SLA may require a service $s_{1}$ to have response time of 15 seconds and sampling rate of $50 \%$ to demonstrate a QoS has been met, with a penalty of $\$ 100$ for exceeding this. 
We say that the benefit is $\$ 100$ for monitoring service $s_{1}$ for response time whilst that response time is less than 15 seconds with a sampling rate of at least 0.5 .

A requirement that the quality $q$ of service $s$ must be at least $q l$ and monitored with a sampling rate at least $s r$ can be represented as $r=(s, q, q l, s r)$, and the requirement $r$ with a fine for non-compliance can be represented as $r p=$ $((s, q, q l, s r) \mapsto$ fine $)$. The quality level $q l$ is a fraction of the required quality level over the ideal quality level that is provided by the service. For example, if the best possible response time of a service is 10 seconds, and the required response time is 20 seconds, then the quality level $q l$ is 0.5 . The fine is the penalty that must be paid if the sampling rate $s r$ and the quality level $q l$ are not met. The set of all requirements with fines is $R P=\{(s, q, q l, s r) \mapsto$ fine : $\mathbb{R} \mid \forall(s, q, q l, s r) \in R\}$, where $R$ is the set of all requirements.

Consider the requirements that the quality $q$ of service $s$ should be monitored all the time and achieve a quality level of 0.75 , with a fine of $\$ 60$ if the quality level goes below 0.75 , and a fine of $\$ 90$ if the quality level goes below 0.5 . This set of requirements with fines can be represented as rps $=\left\{\left(s_{1}, q_{1}, .50,1\right) \mapsto\right.$ $\left.\$ 90,\left(s_{1}, q_{1}, .75,1\right) \mapsto \$ 60,\left(s_{1}, q_{1}, 1,1\right) \mapsto \$ 0\right\}$.

We transform each monitoring requirement describing fines for non-compliance into utility functions describing benefit (utility) for compliance. Since our utilities are cumulative, for $r p=((s, q, q l, s r) \mapsto$ fine $)$, we have the corresponding utility util $(s, q, q l, s r)=(\operatorname{curmax}-R P(s, q, q l, s r))-\sum_{l r \in L R(s, q, s q, s r)}($ curmax$u t i l(l r)$ ), where curmax is the benefit of achieving the quality level $q l$ and sampling rate $s r$, and $L R$ is the set of requirements with a quality level $l r . q l<q l$ or sampling rate $l r . s r<s r, L R(s, q, q l, s r)=\left\{\left(s, q, q l_{1}, s r_{1}\right) \mid \forall\left(s, q, q l_{1}, s r_{1}\right) \in\right.$ $\left.R\left(\left(q l_{1}<q_{1}\right) \wedge\left(s r_{1} \leq s r\right)\right) \vee\left(\left(q l_{1} \leq q_{1}\right) \wedge\left(s r_{1}<s r\right)\right)\right\}$. Therefore, the utility function covering all the monitoring requirements is $U=\{(s, q, q l, s r) \mapsto$ $u t i l(s, q, q l, s r) \mid(s, q, q l, s r) \in R\}$.

For the above set of example requirements rps, we have the following corresponding utilities: $u s=\left\{\left(s_{1}, q_{1}, .50,1\right) \mapsto \$ 0,\left(s_{1}, q_{1}, .75,1\right) \mapsto \$ 30,\left(s_{1}, q_{1}, 1,1\right) \mapsto \$ 60\right\}$. The last element $\left(\left(s_{1}, q_{1}, 1,1\right) \mapsto \$ 60\right)$ of the utility set us is read as "achieving quality level greater than .75 of quality $q_{1}$ for service $s_{1}$ with a sampling rate of 1 is worth an additional $\$ 60$ (relative to the next lower quality level of 0.75 ), since fines of that amount will not have to be paid". The absolute utility for the $q l=1$ level is $\$ 90$, including the utility at the lower quality level (\$30). Designers may also add utilities that are not directly derived from requirements.

Optimizer. The Optimizer takes as input the set of utilities $U$, the set of monitoring configurations with impacts $M C I$, and the ideal service quality levels for all qualities of all services $I Q L=\{(s, q) \mapsto i q l: Q L \mid \forall s \in S, \forall q \in Q\}$, where $i q l$ is the pre-determined best achievable quality level for quality $q$ of service $s$. The Optimizer outputs a monitoring configuration that gives the best utility in terms of meeting requirements and reducing monitoring impacts.

For a monitoring configuration $m c$ with impacts, the Optimizer first obtains the Final Quality Levels $F Q L$ of all $(s, q)$ pairs by subtracting all relevant impacts from their ideal quality levels $I Q L$, i.e., $F Q L(m c)=\{(s, q) \mapsto f q l: Q L \mid i q l=$ $\operatorname{IQL}(s, q)-M C I(m c)(s, q)\}$. 
The utility of the $(s, q)$ pair under monitoring configuration $m c, u(m c)(s, q)$, is the sum of those utilities of $(s, q),(1)$ whose required quality level $q l$ has been met by the pair's final quality level $F Q L(m c)(s, q)$ and $(2)$ whose sampling rate $s r$ is met by at least one monitor setting in $m c$.

Let $R_{(s, q)}$ be the set of all requirements concerning $(s, q)$, i.e., $R_{(s, q)}=\left\{\left(s_{1}, q_{1}\right.\right.$, $\left.q l, s r) \mid\left(s_{1}, q_{1}, q l, s r\right) \in R \wedge s_{1}=s \wedge q_{1}=q\right\}$. Then, $u(m c)(s, q)=$ $\sum_{(s, q, q l, s r) \in R_{(s, q)}}(u \operatorname{til}(m, s, q l, s r) \times a \times b$, where

$a=\left\{\begin{array}{l}1 \text { iff } F Q L(s, q) \geq q l \\ 0 \text { iff } F Q L(s, q)<q l\end{array}\right\}, b=\left\{\begin{array}{l}1 \text { iff } \exists m \in M, m s(m, s, q) \geq s r \\ 0 i f f \nexists m \in M, m s(m, s, q) \geq s r\end{array}\right\}$.

The total utility for the monitoring configuration $m c$ will be the sum of the utilities for all $(s, q)$ pairs under the configuration, $u(m c)$. Let $S Q_{m c}=\{(s, q) \mid \forall$ $(m, s, q, l) \in m c\}$. Then, $u(m c)=\sum_{(s, q) \in S Q_{m c}} u(m c)(s, q)$. The set of all monitoring configurations with utility is, $M C U=\{m c \mapsto u(m c) \mid m c \in M C\}$. A monitoring configuration that gives the highest total utility will be an optimal monitoring configuration, optimal ${ }_{m c} \in\left\{m c_{1} \mid \forall m c_{2} \in M C, M C U\left(m c_{1}\right) \geq\right.$ $\left.M C U\left(m c_{2}\right)\right\}$, which may be applied to the monitors in the system either manually or automatically (if available via methods such as SNMP or WS-Management).

Optimization Complexity. The number of monitoring configurations is the product of the number of monitoring levels $\left(M L_{\text {mcap }}\right)$ for all monitoring capabilities in the system (mcap $\in M C A P)$. The size of this search space is bounded by $\mathcal{O}\left(\operatorname{avg}\left(\left|M L_{\text {mcap }}\right|\right)^{|M C A P|)}\right)$. Since we step through the search space once for each of Impact Analysis and Optimization, this is also the time complexity for our optimization technique. The problem is a combinatorial optimization problem with both overhead (fixed) and instance (variable) costs, a case of an integer fixed charge network flow (FCNFP) problem, demonstrated to be NP-hard 5. We have provided a brute force technique to demonstrate the possible performance and utility benefits of applying optimization. This also provides a baseline of a 'perfect' optimization against which to compare future heuristic algorithms.

\section{Performance Evaluation}

A prototype1 of a travel agency with two web services, five monitors, four quality types, six SLA requirements, four Corporate Governance requirements, and $2.5 \times 10^{5}$ possible monitoring configurations has been implemented to verify our optimization technique and measure possible performance benefits of monitoring optimization. There always exists one or more optimal monitoring configurations (yielding the highest utility) in terms of monitoring coverage and performance. The purpose of these experiments is to discover these optimal monitoring configurations, and compare the utility and performance of each optimal configuration to the utility and performance of each corresponding maximum (un-optimized) monitoring configuration, in which all monitors run at a $100 \%$ sampling rate.

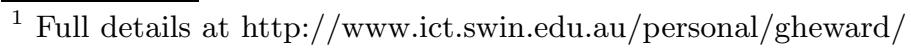


The performance measurements are based on a series of simulated service executions, which use response time measured through real service invocations to determine what the performance will be for a given monitoring configuration.

\subsection{Results}

We report average response times and utilities to demonstrate that system performance has increased whilst monitoring requirements have been met. Utility increased in each experiment, as only the minimum valuable monitoring level was met, allowing for a performance increase which minimised the chance of breaching requirements for response time. Figure 2 shows response time versus load level with unmonitored, maximum, and optimal monitoring configurations. The load levels on the horizontal axis represent the average number of active client requests, and the vertical axis is the average response time over both services.

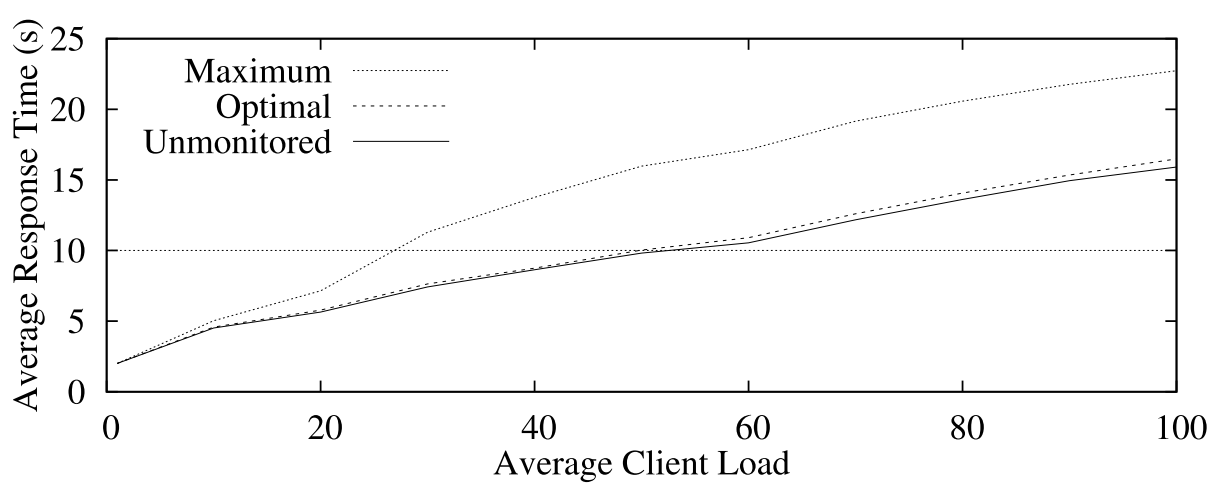

Fig. 2. Performance Evaluation

Optimization reduced the average response time by approximately $30 \%$ from maximum monitoring, and reduced the average response time impact by $80 \%$.

The horizontal line on Figure 2 shows the 10-second boundary, for which penalties apply in the test system. The optimized monitoring configuration stays under this 10-second boundary for approximately twice as long as the maximum monitoring configuration, i.e. the system with optimized monitoring dealt with twice as much load before a penalty would have been paid.

The utility provided by the unmonitored solution was 0 , since no requirements could be verified. The Maximum and Optimal monitoring configurations both included complete monitoring coverage. For each of these two configurations, the utility at or below 10 seconds response time was 4.245 , and the utility above 10 seconds response time was 3.75 . We repeated all experiments with randomly generated utility functions and monitoring impacts ranging from mild (less than $.01 \%$ per monitoring instance on average) to severe (up to $20 \%$ per monitoring instance), under various load levels. The response times for the optimized solutions were on average $40 \%$ lower than maximum monitoring, and the optimized 
response time impacts were on average $70 \%$ lower than maximum monitoring. Utility values were on average $30 \%$ higher for the optimized solutions.

\section{Related Work}

Ranganathan and Dan present a Web Services management system to monitor and reallocate local system resources for services based on comparing their current QoS to their SLAs[6]. Whilst performing system-level administration, this method does not re-configure the web service monitoring system.

Baresi and Guinea present a method for dynamic monitoring of WS-BPEL processes, which uses high level monitoring rules [4]. These Monitoring Rules are used to control the monitoring of each WS-BPEL process. The rules are created with an equivalent of debug levels (one to five), which allows for optimization in terms of performance versus monitoring trade-offs at run-time. The monitoring level must be set for each service as a unit. Rather than assigning a monitoring resolution (debug level) to each individual service in the system, we assign a monitoring resolution to each quality of each service, directly reflecting requirements from SLAs. Furthermore, we enhance the optimization by selecting those monitors which will most efficiently monitor each service.

Overall, there have been numerous efforts for optimizing the QoS of web services and web service compositions, which consider the selection of services in order to optimize QoS. We have discovered no work that optimizes a web services monitoring system by trading off between monitoring costs and benefits, directly translated from SLAs and other requirements for monitoring.

\section{Conclusions and Future Work}

We have presented a framework and techniques for optimizing the configuration of web service monitors, in order to maximise utility for a web services provider.

A prototype instantiation of our proposed framework was used for a series of experiments, which demonstrated that both utility and performance can be improved by optimally configuring a web services monitoring system. Results indicate that the performance impact of web service monitoring can be significantly reduced, whilst meeting monitoring requirements.

We plan to extend the framework and implementation so that run-time properties of the system being monitored are fed back into the monitoring optimization framework, for re-optimization at run-time. We will also develop a heuristic approach to optimization in order to ensure scalability of the technique.

Acknowledgements. This work is supported by the Australian Research Council in collaboration with CA Labs.

\section{References}

1. Heward, G., Müller, I., Han, J., Schneider, J.G., Versteeg, S.: Assessing the performance impact of service monitoring. In: Australian Software Engineering Conference (ASWEC 2010), pp. 192-201 (2010) 
2. Ludwig, H., Dan, A., Kearney, R.: Cremona: An architecture and library for creation and monitoring of ws-agreements. In: International Conference on Service Oriented Computing (ICSOC 2004), vol. 74, pp. 65-74 (2004)

3. Cardellini, V., Casalicchio, E., Grassi, V., Lo Presti, F.: Flow-based service selection for web service composition supporting multiple qos classes. In: International Conference on Web Services (ICWS 2007), pp. 743-750 (2007)

4. Baresi, L., Guinea, S.: Towards dynamic monitoring of ws-bpel processes. In: Benatallah, B., Casati, F., Traverso, P. (eds.) ICSOC 2005. LNCS, vol. 3826, pp. 269-282. Springer, Heidelberg (2005)

5. Stevens, T., Vermeir, J., Leenheer, M.D., Develder, C., Turck, F.D., Dhoedt, B., Demeester, P.: Distributed service provisioning using stateful anycast communications. In: Annual IEEE Conference on Local Computer Networks (LCN 2007), pp. 165-174 (2007)

6. Ranganathan, K., Dan, A.: Proactive management of service instance pools for meeting service level agreements. In: Benatallah, B., Casati, F., Traverso, P. (eds.) ICSOC 2005. LNCS, vol. 3826, pp. 296-309. Springer, Heidelberg (2005) 